\title{
LIFTS OF PROJECTIVE CONGRUENCE GROUPS, II
}

\author{
IAN KIMING
}

(Communicated by Matthew A. Papanikolas)

\begin{abstract}
We continue and complete our previous paper "Lifts of projective congruence groups" concerning the question of whether there exist noncongruence subgroups of $\mathrm{SL}_{2}(\mathbb{Z})$ that are projectively equivalent to one of the groups $\Gamma_{0}(N)$ or $\Gamma_{1}(N)$. A complete answer to this question is obtained: In case of $\Gamma_{0}(N)$ such noncongruence subgroups exist precisely if $N \notin\{3,4,8\}$ and we additionally have either that $4 \mid N$ or that $N$ is divisible by an odd prime congruent to 3 modulo 4 . In case of $\Gamma_{1}(N)$ these noncongruence subgroups exist precisely if $N>4$.

As in our previous paper the main motivation for this question is the fact that the above noncongruence subgroups represent a fairly accessible and explicitly constructible reservoir of examples of noncongruence subgroups of $\mathrm{SL}_{2}(\mathbb{Z})$ that can serve as the basis for experimentation with modular forms on noncongruence subgroups.
\end{abstract}

\section{INTRODUCTION}

Let $N \in \mathbb{N}$ and let $\Gamma$ be one of the standard congruence subgroups $\Gamma_{0}(N), \Gamma_{1}(N)$, or $\Gamma(N)$. Denote by $\bar{\Gamma}$ the image of $\Gamma$ in $\operatorname{PSL}_{2}(\mathbb{Z})$. For $\Gamma_{1}$ a subgroup of $\mathrm{SL}_{2}(\mathbb{Z})$ (of finite index) we say that $\Gamma_{1}$ is a lift of $\bar{\Gamma}$ if $\Gamma_{1}$ projects to $\bar{\Gamma}$ under the canonical homomorphism $\mathrm{SL}_{2}(\mathbb{Z}) \rightarrow \mathrm{PSL}_{2}(\mathbb{Z})$.

In our previous paper 2 we discovered that not only is it possible for a congruence $\bar{\Gamma}$ as above to have a noncongruence lift, i.e., a lift $\Gamma_{1}$ that is not a congruence subgroup, i.e., that does not contain $\Gamma(M)$ for any $M$, but that, in fact, the number of noncongruence lifts appears to usually dominate the number of congruence lifts. Here, "usually" should be taken to mean "apart from the cases where simple obstructions trivially prevent this, and apart from the cases where $N$ is small". However, a number of hard cases were left out of the analysis in [2], and some of the results of that paper depended on machine computations.

The principal interest in these questions lies in the fact that noncongruence lifts of a group $\bar{\Gamma}$ provide relatively easy examples of noncongruence groups, and that, because our approach to these noncongruence lifts is constructive, there is a possibility of studying spaces of modular forms on such noncongruence lifts. Cf. for example the analysis in [2] of spaces of modular forms of weight 3 on the various lifts of the group $\overline{\Gamma_{1}(6)}$. As our knowledge of the arithmetic of modular forms on noncongruence subgroups is still fairly limited compared with the situation for congruence subgroups, having a readily accessible reservoir of examples of noncongruence subgroups is valuable as a tool for exploration and experimentation.

Received by the editors August 10, 2012 and, in revised form, December 21, 2012. 2010 Mathematics Subject Classification. Primary 11F06; Secondary 20H05. 
The purpose of the present paper is to augment the previous paper [2] so as to obtain a complete description of the situation for the above series of groups and for all $N$. We shall use a slightly different method of proof and are in fact able to prove everything from the ground up and also avoid all machine computations. Our results are as follows.

Theorem 1.1. Let $N \in \mathbb{N}$ and write $N=2^{s} p_{1}^{s_{1}} \cdots p_{t}^{s_{t}}$ where the $p_{i}$ are distinct primes, $s_{i} \in \mathbb{N}$, and $s \in \mathbb{Z}_{\geq 0}$.

Then the number of congruence lifts of the subgroup $\overline{\Gamma_{0}(N)} \leq \mathrm{PSL}_{2}(\mathbb{Z})$ is

$$
\left\{\begin{array}{lll}
1, & \text { if } s \leq 1 \text { and } p_{i} \equiv 1 \\
1+2^{s+t}, & \text { if } s \leq 1 \text { and } p_{i} \equiv 3 \quad \text { (4) for all } i, \\
1+2^{\min \{3, s\}+t}, & \text { if } s \geq 2
\end{array}\right.
$$

The number of congruence lifts of the subgroup $\overline{\Gamma_{1}(N)} \leq \mathrm{PSL}_{2}(\mathbb{Z})$ is

$$
\begin{cases}1, & \text { if } N \leq 2 \\ 3, & \text { if } N>2 \text { is odd } \\ 5, & \text { if } N>2 \text { is even }\end{cases}
$$

The number of congruence lifts of the subgroup $\overline{\Gamma(N)} \leq \mathrm{PSL}_{2}(\mathbb{Z})$ is

$$
\begin{cases}1, & \text { if } N=1, \\ 3, & \text { if } N>1 \text { is odd, } \\ 5, & \text { if } N=2, \\ 9, & \text { if } N>2 \text { is even. }\end{cases}
$$

Theorem 1.2. Let $N \in \mathbb{N}$. All lifts of $\overline{\Gamma_{0}(N)} \leq \mathrm{PSL}_{2}(\mathbb{Z})$ are congruence subgroups of $\mathrm{SL}_{2}(\mathbb{Z})$ if and only if either $N \in\{3,4,8\}$ or if $4 \nmid N$ and all odd prime divisors of $N$ are congruent to 1 modulo 4 .

Theorem 1.3. Let $N \in \mathbb{N}$. All lifts of $\overline{\Gamma_{1}(N)} \leq \mathrm{PSL}_{2}(\mathbb{Z})$ are congruence subgroups of $\mathrm{SL}_{2}(\mathbb{Z})$ if and only if $N \leq 4$.

Being complete classifications of the situation regarding the question of existence of noncongruence lifts of $\bar{\Gamma}$ when $\Gamma$ is either $\Gamma_{0}(N)$ or $\Gamma_{1}(N)$, the above theorems obviously deal with some of the remaining, hard cases that were left undecided in our previous paper [2]. These hard cases contain, for instance, all cases of $\Gamma_{1}(N)$ when $N$ is 4 times an odd number $>1$, as well as the cases concerning $\Gamma_{0}(N)$ when $N$ is 4 times an odd number $>1$, all of whose prime divisors are congruent to 1 modulo 4.

Below we shall prove the above theorems by a somewhat different and more general method than in [2], although the basic principles of proof remain the same. Specifically, we shall utilise the paper 3] for information about generators and relations for the group $\bar{\Gamma}$ in the various cases. Combined with the group theoretical analysis in section 2, which leads to a proof of Theorem 1.1] this allows us to prove Theorems 1.2 and 1.3 basically ab initio, i.e., essentially without referring back to the results of 2] (this is apart from certain elementary observations). In particular, this new approach bypasses the need for computation that was necessary in certain cases in the paper [2].

The paper 2] already proved that there are noncongruence lifts of the group $\overline{\Gamma(N)}$ if (and only if) $N>2$. It would have been easy to include the proof of this fact here by using our current approach, but we have chosen not to do so. 


\section{Congruence Lifts}

Theorem 1.1 follows readily by combining Proposition 2.1 and Corollary 2.4 below.

For a finite group $G$ denote as usual by $G^{\prime}$ the derived group of $G$, and by $G^{2}$ the characteristic subgroup of $G$ generated by all squares of elements of $G$. The subgroup $G^{\prime} G^{2}$ is then the largest normal subgroup of $G$ with elementary abelian quotient of 2-power order. (One has in fact $G^{\prime} G^{2}=G^{2}$, since all commutators can be expressed as products of squares. Also, if $G$ is a 2-group, then $G^{\prime} G^{2}$ is the Frattini subgroup of $G$. However, we will not need this information.)

Let us recall Wohlfahrt's notion of the "general level" of a subgroup $\Gamma$ of finite index in $\mathrm{SL}_{2}(\mathbb{Z})$; cf. [7]: the general level of $\Gamma$ is defined to be the least common multiple of all cusp widths where these widths are computed relative to the projective image $\bar{\Gamma} \leq \operatorname{PSL}_{2}(\mathbb{Z})$. That is, the width of a cusp $c$ is the least $n \in \mathbb{N}$ such that

$$
\pm g\left(\begin{array}{ll}
1 & n \\
0 & 1
\end{array}\right) g^{-1} \in \Gamma
$$

where $g \in \mathrm{SL}_{2}(\mathbb{Z})$ is such that $g \infty=c$. Thus, the general level of $\Gamma$ depends only on its projective image $\bar{\Gamma}$.

Proposition 2.1. Let $\Gamma$ be a congruence subgroup of $\mathrm{SL}_{2}(\mathbb{Z})$ of general level $N$.

Then $\Gamma \geq \Gamma(2 N)$. We put $G:=\Gamma / \Gamma(2 N)$ and consider the quotient $G / G^{\prime} G^{2}$ as a vector space over $\mathbb{F}_{2}$. Define:

$$
d:=\operatorname{dim}_{\mathbb{F}_{2}} G / G^{\prime} G^{2} .
$$

Abusing notation and denoting by -1 both the matrix $\left(\begin{array}{cc}-1 & 0 \\ 0 & -1\end{array}\right) \in \mathrm{SL}_{2}(\mathbb{Z})$ as well as its image in $G$, the number of congruence lifts of $\bar{\Gamma}$ is

$$
\begin{cases}1, & \text { if }-1 \in G^{\prime} G^{2}, \\ 1+2^{d-1}, & \text { if }-1 \in \Gamma \text { but }-1 \notin G^{\prime} G^{2}, \\ 1+2^{d}, & \text { if }-1 \notin \Gamma .\end{cases}
$$

Before the proof, we need the following simple lemma from linear algebra.

Lemma 2.2. Let $p$ be a prime, let $d \in \mathbb{N}$ and let $V$ be a vector space of dimension $d$ over $\mathbb{F}_{p}$.

If $0 \neq v \in V$ is a given nonzero vector, then the number of subspaces of $V$ of codimension 1 and not containing $v$ is $p^{d-1}$.

Proof. The statement is obviously true when $d=1$, so we proceed under the assumption that $d>1$.

The subspaces of $V$ of codimension 1 are in $1-1$ correspondence with surjective linear maps $V \rightarrow \mathbb{F}_{p}$ modulo scalars $\neq 0$. Hence the number $A$ of such subspaces is

$$
A=\frac{p^{d}-1}{p-1}=p^{d-1}+\ldots+1
$$

as the cardinality of the dual vector space $V^{*}$ is $\# V^{*}=\# V=p^{d}$.

Now let a nonzero vector $v \in V$ be given. The number $B$ of codimension 1 subspaces of $V$ not containing $v$ is $B=A-C$ where $C$ is the number of codimension 1 subspaces $W$ of $V$ with $v \in W$.

Clearly, $C$ is the number of surjective linear maps $V \rightarrow \mathbb{F}_{p}$ with $v$ in the kernel, counted modulo scalars $\neq 0$. Again, this can be identified as the number of surjective linear maps $V /\langle v\rangle \rightarrow \mathbb{F}_{p}$ modulo scalars $\neq 0$. As $V /\langle v\rangle$ has dimension $d-1$, it 
follows from the first part of the proof that

But then,

$$
C=\frac{p^{d-1}-1}{p-1} .
$$

$$
B=A-C=p^{d-1} .
$$

Proof of Proposition 2.1. As $\Gamma$ is a congruence subgroup of $\mathrm{SL}_{2}(\mathbb{Z})$ of general level $N$, we know by Proposition 3 of 2 that $\Gamma$ contains $\Gamma(2 N)$. (This result is essentially due to Wohlfahrt [7, cf. the discussion after Proposition 1 of [2].) Furthermore, if $\Gamma_{1}$ is a congruence lift of $\bar{\Gamma}$, then $\Gamma_{1}$ also has general level $N$ and hence also contains $\Gamma(2 N)$. Also, for any lift $\Gamma_{1}$ of $\bar{\Gamma}$ we must necessarily have $\left\langle\Gamma_{1},-1\right\rangle=\langle\Gamma,-1\rangle$ since $\langle-1\rangle$ is the kernel of the projection map $\mathrm{SL}_{2}(\mathbb{Z}) \rightarrow \mathrm{PSL}_{2}(\mathbb{Z})$.

Suppose first that $-1 \in \Gamma$. It then follows from the above that congruence lifts of $\bar{\Gamma}$ correspond one-to-one to subgroups $U$ of $G:=\Gamma / \Gamma(2 N)$ such that $G$ is generated by $U$ and the image of -1 . Such a subgroup $U$ is then necessarily of index $\leq 2$ in $G$ and hence is normal in $G$ and contains $G^{\prime} G^{2}$. Thus, these subgroups $U$ are in one-to-one correspondence with subspaces $W$ of the $\mathbb{F}_{2}$-vector space $V:=G / G^{\prime} G^{2}$ such that $V$ is generated by $W$ and the image $v$ of -1 . If $v=0$, i.e., if $-1 \in G^{\prime} G^{2}$, there is only one such subspace, namely $V$ itself. On the other hand, if $-1 \notin G^{\prime} G^{2}$, then $v \neq 0$, and apart from $V$ itself the possibilities for the subspaces $W$ are the codimension 1 subspaces of $V$ not containing $v$. By Lemma 2.2 the number of the latter subspaces is $2^{d-1}$ where $d:=\operatorname{dim}_{\mathbb{F}_{2}} G / G^{\prime} G^{2}$; observe that, because of the condition $-1 \notin G^{\prime} G^{2}$, we certainly have $d \geq 1$ in this case.

We have now established the proposition in case $-1 \in \Gamma$.

Suppose then that $-1 \notin \Gamma$ and consider the group $\widetilde{\Gamma}:=\langle\Gamma,-1\rangle$. Then $\widetilde{\Gamma}$ is a congruence subgroup with image $\bar{\Gamma}$ in $\operatorname{PSL}_{2}(\mathbb{Z})$ and hence also has general level $N$. As now $-1 \in \widetilde{\Gamma}$, and as congruence lifts of $\bar{\Gamma}$ and of $\overline{\widetilde{\Gamma}}$ are trivially the same things, we can now apply the previous discussion to $\widetilde{\Gamma}$. So, we consider the group $\widetilde{G}:=\widetilde{\Gamma} / \Gamma(2 N)$ and the dimension $\tilde{d}:=\operatorname{dim}_{\mathbb{F}_{2}} \widetilde{G} / \widetilde{G}^{\prime} \widetilde{G}^{2}$. Clearly, $\widetilde{G} \cong\langle-1\rangle \times G$, whence we see that $\tilde{d}=d+1$. As $\widetilde{G}^{\prime} \widetilde{G}^{2}=G^{\prime} G^{2}$, the hypothesis $-1 \notin \Gamma$ implies $-1 \notin \widetilde{G}^{\prime} \widetilde{G}^{2}$. Hence the previous discussion implies the statement of the proposition in the present case.

Proposition 2.3. (i) Let $p$ be an odd prime and let $s \in \mathbb{N}$.

If $G_{0}:=\Gamma_{0}\left(p^{s}\right) / \Gamma\left(p^{s}\right)$, then $G_{0} /\left(G_{0}^{\prime} G_{0}^{2}\right)$ is cyclic of order 2 . Further, $-1 \in G_{0}^{\prime} G_{0}^{2}$ if and only if $p \equiv 1$ (4).

If $G_{1}:=\Gamma_{1}\left(p^{s}\right) / \Gamma\left(p^{s}\right)$, then $G_{1}=G_{1}^{\prime} G_{1}^{2}$.

We have $-1 \in \Gamma_{0}\left(p^{s}\right)$, but $-1 \notin \Gamma_{1}\left(p^{s}\right), \Gamma\left(p^{s}\right)$.

(ii) Let $s \in \mathbb{Z}_{\geq 0}$.

If $G_{0}:=\Gamma_{0}\left(2^{\bar{s}}\right) / \Gamma\left(2^{s+1}\right)$, then

$$
G_{0} /\left(G_{0}^{\prime} G_{0}^{2}\right) \cong \begin{cases}(\mathbb{Z} / 2)^{s+1} & \text { if } s \leq 2 \\ (\mathbb{Z} / 2)^{4} & \text { if } s \geq 3 .\end{cases}
$$

We have $-1 \in \Gamma_{0}\left(2^{s}\right)$, but $-1 \in G_{0}^{\prime} G_{0}^{2}$ if and only if $s \leq 1$. If $G_{1}:=\Gamma_{1}\left(2^{s}\right) / \Gamma\left(2^{s+1}\right)$, then

$$
G_{1} /\left(G_{1}^{\prime} G_{1}^{2}\right) \cong \begin{cases}\mathbb{Z} / 2 & \text { if } s=0 \\ (\mathbb{Z} / 2)^{2} & \text { if } s \geq 1 .\end{cases}
$$

We have $-1 \in \Gamma_{1}\left(2^{s}\right)$ if and only if $-1 \in G_{1}^{\prime} G_{1}^{2}$ if and only if $s \leq 1$. 
Finally, if $G:=\Gamma\left(2^{s}\right) / \Gamma\left(2^{s+1}\right)$, then

$$
G /\left(G^{\prime} G^{2}\right) \cong \begin{cases}\mathbb{Z} / 2 & \text { if } s=0, \\ (\mathbb{Z} / 2)^{3} & \text { if } s \geq 1,\end{cases}
$$

and we have $-1 \in \Gamma\left(2^{s}\right)$ if and only if $s \leq 1$, and $-1 \in G^{\prime} G^{2}$ if and only if $s=0$.

Proof. For $N \in \mathbb{N}$ we have in general that $\mathrm{SL}_{2}(\mathbb{Z}) / \Gamma(N) \stackrel{\sim}{\longrightarrow} \mathrm{SL}_{2}(\mathbb{Z} / N)$ with the isomorphism induced by reducing matrix entries modulo $N$. Also, we have in $\Gamma_{0}(N)$ a normal series $\Gamma_{0}(N) \unrhd \Gamma_{1}(N) \unrhd \Gamma(N)$ with successive quotients:

$$
\Gamma_{0}(N) / \Gamma_{1}(N) \cong(\mathbb{Z} / N)^{\times}
$$

generated by matrices modulo $N$ of the shape $\left(\begin{array}{ll}a & 0 \\ 0 & b\end{array}\right)$ where $a$ and $b$ are integers with $a b \equiv 1(N)$, as well as

generated by the matrix $\left(\begin{array}{ll}1 & 1 \\ 0 & 1\end{array}\right)$ modulo $N$.

$$
\Gamma_{1}(N) / \Gamma(N) \cong \mathbb{Z} / N
$$

(i) As $p$ is odd, the group $\left(\mathbb{Z} / p^{s}\right)^{\times}$is cyclic of order $p^{s-1}(p-1)$. If $a$ is a generator and $b$ is such that $a b \equiv 1\left(p^{s}\right)$, the quotient $\Gamma_{0}\left(p^{s}\right) / \Gamma_{1}\left(p^{s}\right)$ is then generated by the image of

$$
\xi:=\left(\begin{array}{ll}
a & 0 \\
0 & b
\end{array}\right) \quad\left(\bmod p^{s}\right) .
$$

On the other hand, $\Gamma_{1}\left(p^{s}\right) / \Gamma\left(p^{s}\right)$ is generated modulo $p^{s}$ by

$$
\tau:=\left(\begin{array}{ll}
1 & 1 \\
0 & 1
\end{array}\right) \text {. }
$$

Now, since $p$ is odd we see that $\tau$ is a suitable power of $\tau^{2}$ so that $\left\langle\xi^{2}, \tau^{2}\right\rangle=$ $\left\langle\xi^{2}, \tau\right\rangle$. But $\left\langle\xi^{2}, \tau\right\rangle$ is normal in $G_{0}$ with quotient cyclic of order 2 . It follows that $G_{0}^{\prime} G_{0}^{2}=\left\langle\xi^{2}, \tau^{2}\right\rangle=\left\langle\xi^{2}, \tau\right\rangle$ and hence that $G_{0} /\left(G_{0}^{\prime} G_{0}^{2}\right)$ is cyclic of order 2 , as claimed. We also see that $-1 \in G_{0}^{\prime} G_{0}^{2}=\left\langle\xi^{2}, \tau\right\rangle$ if and only if -1 is a square modulo $p$ which happens precisely if $p \equiv 1$ (4).

As $s \geq 1$ and $p$ is odd, the group $\Gamma_{1}\left(p^{s}\right)$ does not contain -1 . Since $G_{1}$ is cyclic of odd order $p^{s}$ we have $G_{1}=G_{1}^{\prime} G_{1}^{2}$.

(ii) We will be considering the normal series

$$
\Gamma_{0}\left(2^{s}\right) \unrhd \Gamma_{1}\left(2^{s}\right) \unrhd \Gamma\left(2^{s}\right) \unrhd \Gamma\left(2^{s+1}\right)
$$

as well as the corresponding normal series in $G_{0}=\Gamma_{0}\left(2^{s}\right) / \Gamma\left(2^{s+1}\right)$.

If $s=0$, the three groups $G_{0}, G_{1}$, and $G$ coincide and are isomorphic to $\mathrm{SL}_{2}(\mathbb{Z}) / \Gamma(2) \cong \mathrm{SL}_{2}\left(\mathbb{F}_{2}\right) \cong S_{3}$. It follows that $G /\left(G^{\prime} G^{2}\right)$ is cyclic of order 2 in this case. We have $-1 \in \Gamma(2)$ and hence the image of this element is trivial in $G$.

We may now assume $s \geq 1$ for the rest of the proof.

Then the quotient $G:=\Gamma\left(2^{s}\right) / \Gamma\left(2^{s+1}\right)$ has order 8 , and one checks that it is in fact isomorphic to $(\mathbb{Z} / 2)^{3}$ and generated by the following matrices modulo $2^{s+1}$ :

$$
\alpha:=\left(\begin{array}{cc}
1 & 2^{s} \\
0 & 1
\end{array}\right), \beta:=\left(\begin{array}{cc}
1+2^{s} & 2^{s} \\
0 & 1+2^{s}
\end{array}\right), \gamma:=\left(\begin{array}{cc}
1 & 0 \\
2^{s} & 1
\end{array}\right) .
$$

It follows that $G^{\prime} G^{2}=1$ and that $-1 \notin G^{\prime} G^{2}$.

The quotient $\Gamma_{1}\left(2^{s}\right) / \Gamma\left(2^{s}\right)$ is generated by the image of the matrix

$$
\tau:=\left(\begin{array}{ll}
1 & 1 \\
0 & 1
\end{array}\right)
$$

so that $\tau^{2^{s}}=\alpha$ in $G$. One computes that $\tau \alpha=\alpha \tau, \tau \beta=\beta \tau$, and that

$$
\tau \gamma \tau^{-1}=\beta \gamma .
$$

We see that $G_{1} /\left\langle\tau^{2}, \beta\right\rangle$ is isomorphic to $(\mathbb{Z} / 2)^{2}$, generated by the images of $\tau$ and $\gamma$. Hence this quotient is an elementary abelian 2-group, whence $\left\langle\tau^{2}, \beta\right\rangle \geq G_{1}^{\prime} G_{1}^{2}$. 
On the other hand, $\beta \in G_{1}^{\prime}$ by the above. Hence $G_{1}^{\prime} G_{1}^{2}=\left\langle\tau^{2}, \beta\right\rangle$. We have $-1 \in \Gamma_{1}\left(2^{s}\right)$ if and only if $s \leq 1$. When $s=1$ we do in fact have $-1 \in G_{1}^{\prime} G_{1}^{2}$ as this element in this case coincides with $\alpha \beta=\tau^{2} \beta$.

It now remains to deal with the group $G_{0}$ for $s \geq 1$.

Assume first that in fact $s \geq 3$. Then $\Gamma_{0}\left(2^{s}\right) / \Gamma_{1}\left(2^{s}\right) \cong\left(\mathbb{Z} / 2^{s}\right)^{\times} \cong \mathbb{Z} / 2 \times \mathbb{Z} / 2^{s-2}$ generated by the images of the following matrices modulo $2^{s+1}$ :

$$
\left(\begin{array}{cc}
-1 & 0 \\
0 & -1
\end{array}\right) \text { and } \xi:=\left(\begin{array}{ll}
a & 0 \\
0 & b
\end{array}\right)
$$

where we have chosen $a$ such that $a$ has order $2^{s-2}$ in $\left(\mathbb{Z} / 2^{s}\right)^{\times}$, generating the second factor in the above decomposition (for instance, we may choose $a:=5$ ), and $b$ such that

$$
a b \equiv 1 \quad\left(2^{s+1}\right) .
$$

One then checks that $\xi$ commutes with $\alpha, \beta, \gamma$ above, and that

$$
[\xi, \tau]:=\xi \tau \xi^{-1} \tau^{-1}=\tau^{a^{2}-1} \in\left\langle\tau^{2}\right\rangle .
$$

It can then be concluded that $G_{0} /\left\langle\xi^{2}, \tau^{2}, \beta\right\rangle$ is isomorphic to $(\mathbb{Z} / 2)^{4}$ with the quotient generated by the images of $-1, \xi, \tau, \gamma\left(\right.$ recall that $\left.\tau^{2^{s}}=\alpha\right)$. In particular, this quotient is an elementary abelian 2-group, and so (as $\beta$ is a commutator) we can deduce that $G_{0}^{\prime} G_{0}^{2}=\left\langle\xi^{2}, \tau^{2}, \beta\right\rangle$ and that $-1 \notin G_{0}^{\prime} G_{0}^{2}$.

If $s=2$, the difference with the case $s \geq 3$ is only that $\Gamma_{0}\left(2^{s}\right) / \Gamma_{1}\left(2^{s}\right) \cong\left(\mathbb{Z} / 2^{s}\right)^{\times}$ is now cyclic of order 2 , generated by -1 . The conclusions in this case then follow in the same way as in the case $s \geq 3$.

When $s=1$ the groups $G_{0}$ and $G_{1}$ coincide so that we have already discussed this case. In particular, we have $-1 \in G_{0}^{\prime} G_{0}^{2}$ when $s=1$.

Corollary 2.4. Let $N \in \mathbb{N}$ and write $N=2^{s} p_{1}^{s_{1}} \cdots p_{t}^{s_{t}}$ where the $p_{i}$ are distinct primes, $s_{i} \in \mathbb{N}$, and $s \in \mathbb{Z}_{\geq 0}$.

If $G_{0}$ denotes the group $G_{0}:=\Gamma_{0}(N) / \Gamma(2 N)$, then

$$
G_{0} /\left(G_{0}^{\prime} G_{0}^{2}\right) \cong(\mathbb{Z} / 2)^{\min \{4, s+1\}+t} .
$$

We have $-1 \in \Gamma_{0}(N)$, but $-1 \in G_{0}^{\prime} G_{0}^{2}$ if and only if $s \leq 1$ and $p_{i} \equiv 1$ (4) for all $i$.

If $G_{1}$ denotes the group $G_{1}:=\Gamma_{1}(N) / \Gamma(2 N)$, then

$$
G_{1} /\left(G_{1}^{\prime} G_{1}^{2}\right) \cong(\mathbb{Z} / 2)^{\min \{2, s+1\}} .
$$

We have $-1 \in \Gamma_{1}(N)$ if and only if $-1 \in G_{1}^{\prime} G_{1}^{2}$ if and only if $s \leq 1$ and $t=0$, i.e., if and only if $N \leq 2$.

If $G$ denotes the group $G:=\Gamma(N) / \Gamma(2 N)$, then

$$
G /\left(G^{\prime} G^{2}\right) \cong \begin{cases}\mathbb{Z} / 2, & \text { if } s=0, \\ (\mathbb{Z} / 2)^{3}, & \text { if } s \geq 1 .\end{cases}
$$

We have $-1 \in \Gamma(N)$ if and only if $s \leq 1$ and $t=0$, i.e., if and only if $N \leq 2$. Furthermore, $-1 \in G^{\prime} G^{2}$ if and only if $s=0$.

Proof. We have a natural isomorphism:

$$
\mathrm{SL}_{2}(\mathbb{Z}) / \Gamma(2 N) \cong \mathrm{SL}_{2}(\mathbb{Z} /(2 N)) \cong \mathrm{SL}_{2}\left(\mathbb{Z} / 2^{s+1}\right) \times \mathrm{SL}_{2}\left(\mathbb{Z} / p_{1}^{s_{1}}\right) \times \cdots \times \mathrm{SL}_{2}\left(\mathbb{Z} / p_{t}^{s_{t}}\right)
$$

given in concrete terms as

$$
A \mapsto(A \quad(\bmod 2 N)) \leftrightarrow\left(\left(A \quad\left(\bmod 2^{s+1}\right)\right),\left(A \quad\left(\bmod p_{1}^{s_{1}}\right)\right), \ldots,\left(A \quad\left(\bmod p_{t}^{s_{t}}\right)\right)\right)
$$

for matrices $A \in \mathrm{SL}_{2}(\mathbb{Z})$ (cf. for instance Lemma 4.2.3 of [4]). 
Under this isomorphism, the subgroup $\Gamma_{0}(N) / \Gamma(2 N) \leq \mathrm{SL}_{2}(\mathbb{Z}) / \Gamma(2 N)$ clearly injects into the subgroup

$$
\Gamma_{0}\left(2^{s}\right) / \Gamma\left(2^{s+1}\right) \times \Gamma_{0}\left(p_{1}^{s_{1}}\right) / \Gamma\left(p_{1}^{s_{1}}\right) \times \cdots \times \Gamma_{0}\left(p_{t}^{s_{t}}\right) / \Gamma\left(p_{t}^{s_{t}}\right)
$$

of

$$
\begin{aligned}
& \mathrm{SL}_{2}(\mathbb{Z}) / \Gamma\left(2^{s+1}\right) \times \mathrm{SL}_{2}(\mathbb{Z}) / \Gamma\left(p_{1}^{s_{1}}\right) \times \cdots \times \mathrm{SL}_{2}(\mathbb{Z}) / \Gamma\left(p_{t}^{s_{t}}\right) \\
\cong & \mathrm{SL}_{2}\left(\mathbb{Z} / 2^{s+1}\right) \times \mathrm{SL}_{2}\left(\mathbb{Z} / p_{1}^{s_{1}}\right) \times \cdots \times \mathrm{SL}_{2}\left(\mathbb{Z} / p_{t}^{s_{t}}\right) .
\end{aligned}
$$

That this map is surjective and hence an isomorphism follows for instance by comparison of orders.

We observe that a similar remark holds for the quotients $\Gamma_{1}(N) / \Gamma(2 N)$ and $\Gamma(N) / \Gamma(2 N)$, and that these decompositions are obviously compatible with formation of the characteristic subgroups $G^{\prime} G^{2}$, etc. Thus the claims of the corollary are seen to follow immediately from Proposition 2.3.

\section{Proofs of Theorems 1.2 and 1.3}

Denote by $\bar{\Gamma}$ either one of the groups $\overline{\Gamma_{0}(N)}$ and $\overline{\Gamma_{1}(N)}$. The proofs follow the same general strategy as in 2]: Using information about a presentation of $\bar{\Gamma}$ in terms of generators and relations, we obtain via Lemma 4 of 2 the total number of lifts of $\bar{\Gamma}$ to $\mathrm{SL}_{2}(\mathbb{Z})$. Comparing this with the information given by Theorem 1.1 above we can decide whether all lifts are congruence subgroups.

As far as a presentation of $\overline{\Gamma_{0}(N)}$ is concerned, the paper 2 cited the results of Chuman in 1. It has since come to our attention that Chuman's paper in fact contains errors; cf. [5]. However, the derivation of the results of [2] did not depend in any way on Chuman's paper.

We shall base our discussion here on Kulkarni's paper 3. Let us recall some consequences of the principal results of that paper: First, the paper describes the group $\bar{\Gamma}$ (in fact, any subgroup of finite index in $\mathrm{PSL}_{2}(\mathbb{Z})$ ) by certain combinatorial objects called generalised Farey sequences. We will not describe these here except to say that such a sequence contains certain numbers $a, b$, and $r$ of "even", "odd", and "pairs of free" "intervals", respectively, that can be used to display $\bar{\Gamma}$ as given in terms of $a+b+r$ generators; cf. Theorem 6.1 of 3 . These generators are such that $a$ of them have order $2, b$ have order 3 , the remaining $r$ are of infinite order, and there are no further relations between the generators. In other words, a presentation of $\bar{\Gamma}$ is given in terms of generators $\bar{g}_{1}, \ldots, \bar{g}_{a+b+r}$ with the following relations:

$$
\bar{g}_{1}^{2}=\ldots=\bar{g}_{a}^{2}=1, \bar{g}_{a+1}^{3}=\ldots=\bar{g}_{a+b}^{3}=1 .
$$

The numbers $a, b$, and $r$ are determined as follows. We have $a=e_{2}, b=e_{3}$, the number of conjugacy classes of subgroups of $\bar{\Gamma}$ of order 2 and 3, respectively; cf. sections (7.1), (7.2) of [3]. Furthermore, the number $r$ is given as

$$
r=\frac{1}{6}\left(d-3 e_{2}-4 e_{3}\right)+1,
$$

where $d$ is the index $\left[\mathrm{PSL}_{2}(\mathbb{Z}): \bar{\Gamma}\right]$; cf. equation (7.1.2) of $[3]$. 
3.1. Proof of Theorem 1.2 , Specialising the above to the case $\bar{\Gamma}=\overline{\Gamma_{0}(N)}$, we have

$$
d=N \prod_{p \mid N}\left(1+\frac{1}{p}\right)
$$

and the determination of the numbers $e_{2}$ and $e_{3}$ is well known; cf. 4, $\S 4.2$, for instance.

The cases left undecided by Theorem 2 of [2] are those where $N$ is 3 , 4, or 8 times an odd number greater than 1 all of whose prime divisors are congruent to 1 modulo 4. Thus, we could limit ourselves to discussing these remaining cases.

However, part of the proof of Theorem 2 of [2] depended on (machine) computations and we want to show here that these can all be avoided. Hence, we will use only the following two results from [2]: First, if $p$ is a prime greater than 3 but congruent to 3 modulo 4 , then there exist noncongruence lifts of $\overline{\Gamma_{0}(p)}$; this is in contrast to the situation for $\overline{\Gamma_{0}(3)}$ that has precisely 3 lifts, all of which are congruence. Cf. Lemma 30 of [2]. The proof utilized Rademacher's presentation of $\overline{\Gamma_{0}(p)}$ for $p$ prime as given in [6].

Second, if $4 \nmid N$ and all odd prime divisors of $N$ are congruent to 1 modulo 4 , then all lifts of $\overline{\Gamma_{0}(N)}$ are congruence; cf. part $(i)$ of Theorem 2 of [2]. The proof consists of a simple observation that in this case, any lift necessarily contains -1 and hence actually equals $\Gamma_{0}(N)$.

We will also utilize the following simple observation (cf. Lemma 5 of [2]):

Lemma 3.1. Suppose that $\Gamma_{1}$ and $\Gamma_{2}$ are subgroups of $\mathrm{SL}_{2}(\mathbb{Z})$ with $\Gamma_{2} \subseteq \Gamma_{1}$. If there exists a noncongruence lift of $\overline{\Gamma_{1}}$, then $\overline{\Gamma_{2}}$ also has a noncongruence lift.

The lemma follows since the pre-image of $\overline{\Gamma_{2}}$ inside a noncongruence lift of $\overline{\Gamma_{1}}$ must obviously necessarily be a noncongruence subgroup of $\mathrm{SL}_{2}(\mathbb{Z})$.

Using Lemma 3.1 together with the starting points described above, one checks that in order to prove Theorem 1.2 it suffices to show:

- If $N \in\{4,8\}$, all lifts of $\overline{\Gamma_{0}(N)}$ are congruence,

- If $N \in\{6,9,16\}$, there are noncongruence lifts of $\overline{\Gamma_{0}(N)}$,

- If $N$ is 3 or 4 times an odd number $>1$ all of whose prime divisors are congruent to 1 modulo 4 , there are noncongruence lifts of $\overline{\Gamma_{0}(N)}$.

When $N=4,6,8,9,16$ one finds $e_{2}=e_{3}=0$ and $d=6,12,12,12,24$, respectively, so that $\overline{\Gamma_{0}(N)}$ is generated in these cases by $r=2,3,3,3,5$ elements with no relations, respectively. Thus, the total number of lifts of $\overline{\Gamma_{0}(N)}$ not containing -1 is $2^{2}, 2^{3}, 2^{3}, 2^{3}, 2^{5}$, respectively; cf. Lemma 4 of 2 . On the other hand, by Theorem 1.1 the number of congruence lifts of $\overline{\Gamma_{0}(N)}$ not containing -1 is $2^{2}, 2^{2}, 2^{3}, 2,2^{3}$, respectively for these cases. It follows that when $N \in\{4,8\}$ all lifts of $\overline{\Gamma_{0}(N)}$ are congruence, whereas there are noncongruence lifts when $N \in\{6,9,16\}$.

Next suppose that $N=4 \cdot p_{1}^{s_{1}} \cdots p_{t}^{s_{t}}$ where $t \geq 1$ and the distinct prime divisors $p_{i}$ are all congruent to 1 modulo 4 . In this case, $e_{2}=e_{3}=0$, and

$$
d=6 \cdot \prod_{i=1}^{t} p_{i}^{s_{i}-1}\left(p_{i}+1\right),
$$

whereas the number of congruence lifts of $\overline{\Gamma_{0}(N)}$ not containing -1 in this case is $2^{2+t}$, by Theorem 1.1. But the total number of lifts not containing -1 is $2^{d / 6+1}$. 
Since $d / 6+1 \geq 1+6^{t}$ which is certainly greater than $2+t$, we conclude that there are in fact noncongruence lifts of $\overline{\Gamma_{0}(N)}$ in this case.

Suppose then that $N=3 \cdot p_{1}^{s_{1}} \cdots p_{t}^{s_{t}}$ where $t \geq 1$ and the distinct prime divisors $p_{i}$ are all congruent to 1 modulo 4 , and that, additionally, $p_{i} \equiv-1$ (3) for at least one $i$. Then $e_{2}=e_{3}=0$,

$$
d=4 \cdot \prod_{i=1}^{t} p_{i}^{s_{i}-1}\left(p_{i}+1\right),
$$

and the total number of lifts of $\overline{\Gamma_{0}(N)}$ not containing -1 is $2^{d / 6+1}$. As the number of congruence lifts not containing -1 is $2^{t}$ by Theorem 1.1 one verifies again that there are noncongruence lifts of $\overline{\Gamma_{0}(N)}$ in this case.

Suppose then finally that $N=3 \cdot p_{1}^{s_{1}} \cdots p_{t}^{s_{t}}$ where $t \geq 1$ and the distinct prime divisors $p_{i}$ are all congruent to 1 modulo 12. Then $e_{2}=0$, but $e_{3}=2^{t}$. Again,

$$
d=4 \cdot \prod_{i=1}^{t} p_{i}^{s_{i}-1}\left(p_{i}+1\right)
$$

but now

$$
r=1+\frac{2}{3}\left(-2^{t}+\prod_{i=1}^{t} p_{i}^{s_{i}-1}\left(p_{i}+1\right)\right) .
$$

Again the number of congruence lifts not containing -1 is $2^{t}$ and the question is whether $2^{r}>2^{t}$. An elementary computation shows this to be the case, and hence the conclusion is again that there are noncongruence lifts of $\overline{\Gamma_{0}(N)}$ in this case.

3.2. Proof of Theorem 1.3. If $N \leq 3$, then $\overline{\Gamma_{1}(N)}=\overline{\Gamma_{0}(N)}$ and hence (by the already proved Theorem 1.2 ) all lifts of $\overline{\Gamma_{1}(N)}$ are congruence.

Assume then that $N \geq 4$ from now on. One then has $e_{2}=e_{3}=0$. Further, the index $d=\left[\mathrm{PSL}_{2}(\mathbb{Z}): \overline{\Gamma_{1}(N)}\right]$ is

$$
d=\frac{N^{2}}{2} \prod_{p \mid N}\left(1-\frac{1}{p^{2}}\right)
$$

and it follows that $\overline{\Gamma_{1}(N)}$ is generated by

$$
r=1+\frac{N^{2}}{12} \prod_{p \mid N}\left(1-\frac{1}{p^{2}}\right)
$$

elements with no relations.

Thus, the total number of lifts of $\overline{\Gamma_{1}(N)}$ is $1+2^{r}$. The question then becomes whether this number exceeds the number of congruence lifts as given by Theorem 1.1

When $N=4$ we have $r=2$ and so $1+2^{r}=5$, which by Theorem 1.1 is precisely the number of congruence lifts. Hence all lifts are congruence when $N=4$.

Suppose then that $N \geq 5$. Then $N$ is divisible by either $6,8,9$ or a prime $p \geq 5$. Now, when $N$ equals $6,8,9$, or a prime $p \geq 5$, we find that $r$ is $3,5,7$, or $\frac{p^{2}+11}{12}$, respectively, and so we see in each case that $1+2^{r}$ exceeds the number of congruence lifts given by Theorem 1.1. Thus there are noncongruence lifts in each of these cases. 
By Lemma 3.1 we can then conclude the existence of noncongruence lifts of $\overline{\Gamma_{1}(N)}$ whenever $N$ is divisible by $6,8,9$, or by a prime $p \geq 5$.

Theorem 1.3 is proved.

\section{REFERENCES}

[1] Yasuhiro Chuman, Generators and relations of $\Gamma_{0}(N)$, J. Math. Kyoto Univ. 13 (1973), 381390. MR0348001 (50 \#499)

[2] Ian Kiming, Matthias Schütt, and Helena A. Verrill, Lifts of projective congruence groups, J. Lond. Math. Soc. (2) 83 (2011), no. 1, 96-120, DOI 10.1112/jlms/jdq062. MR.2763946 (2011m:11082)

[3] Ravi S. Kulkarni, An arithmetic-geometric method in the study of the subgroups of the modular group, Amer. J. Math. 113 (1991), no. 6, 1053-1133, DOI 10.2307/2374900. MR.1137534 (92i:11046)

[4] Toshitsune Miyake, Modular forms, Springer-Verlag, Berlin, 1989. Translated from the Japanese by Yoshitaka Maeda. MR:1021004 (90m:11062)

[5] Antonio Lascurain Orive, Some presentations for $\bar{\Gamma}_{0}(N)$, Conform. Geom. Dyn. 6 (2002), 33-60 (electronic), DOI 10.1090/S1088-4173-02-00073-5. MR1948848(2004a:11031)

[6] H. Rademacher: Über die Erzeugenden von Kongruenzuntergruppen der Modulgruppe, Abh. Math. Sem. Univ. Hamburg 7 (1929), 134-148.

[7] Klaus Wohlfahrt, An extension of F. Klein's level concept, Illinois J. Math. 8 (1964), 529-535. MR0167533(29 \#4805)

Department of Mathematics, University of Copenhagen, Universitetsparken 5, DK2100 Copenhagen $\varnothing$, Denmark

E-mail address: kiming@math.ku.dk 